\title{
FIXED POINTS OF WEAKLY INWARD 1-SET-CONTRACTION MAPPINGS
}

\author{
Huagui Duan, Shaoyuan Xu, and Guozhen Li
}

\begin{abstract}
In this paper, we introduce a fixed point index of weakly inward 1-set-contraction mappings. With the aid of the new index, we obtain some new fixed point theorems, nonzero fixed point theorems and multiple positive fixed points for this class of mappings. As an application of nonzero fixed point theorems, we discuss an eigenvalue problem.
\end{abstract}

\section{Introduction}

Since Halpern and Bergman [9] introduced the conception of inward mapping, many results have appeared in the literatures concerning inward and weakly inward mapping in Halpern's sense (cf. [2], [4], [5], [6], [7], [10], [11], [12], [17], [20], [22], [21], [23]). It is well known that many fixed point theorems for maps involving cones are easily proved if there is a theory of fixed point index for the class of mappings involved.

In 1990, the fixed point index of weakly inward maps that do not necessarily take their values in the cone is defined on compact convex sets [20]. Lan and Webb [11] has introduced a fixed point index for more general mappings of condensing type that satisfy a weaker inwardness condition called generalized inward. On the other hand, Lan and Webb [12] has also studied the class of A-proper mapping and defined a fixed point index for weakly inward mappings.

It is the purpose of this paper to introduce a fixed point index of weakly inward 1-set-contraction mappings by virtue of some results established in Lan and Webb [11]. With the aid of the new index, we obtain some new fixed point theorems, nonzero fixed point theorems and multiple positive fixed points for this class of mappings. As an application of nonzero fixed point theorems, we discuss an eigenvalue problem. It should be noted that this class of 1set-contraction mappings includes self-maps defined on a closed convex set,

\footnotetext{
Received April 8, 2007; Revised November 8, 2007.

2000 Mathematics Subject Classification. 28A78, 28A80.

Key words and phrases. fixed point index, weakly inward 1-set-contraction mapping, fixed

Partially supported by NNSF(No:10801079), RFDP of MOE of China and Nankai Uni-

Partially supported by National Natural Science Foundation of China (No. 10461007).
} point. versity. 
condensing maps, nonexpansive maps, semicontractive maps, LANE maps and others (also see [17]). So our results are generalizations and improvements of the recent results obtained by many authors.

\section{A fixed point index of weakly inward 1-set-contraction mappings}

Let $X$ be a real Banach space. A continuous bounded map $A: \operatorname{dom}(\mathrm{A}) \subset$ $\mathrm{X} \rightarrow \mathrm{X}$ is said to be $k$-set-contraction if $\alpha(A(D)) \leq k \alpha(D)$ for each bounded set $D \subset \operatorname{dom}(\mathrm{A})$ and $k \in R^{+}=[0,+\infty)$, where $\alpha(D)$ is the measure of noncompactness of $D$ defined by

$$
\alpha(D)=\inf \left\{d>0: D=\bigcup_{i=1}^{m} D_{i} \text { for some } m \in N^{+} \text {and } \operatorname{diam}\left(\mathrm{D}_{\mathrm{i}}\right) \leq \mathrm{d}\right\},
$$

here, $N^{+}$denotes the set of all positive integers. If $k \in[0,1)$, then $A$ is called a strict-set-contraction. If $\alpha(A(D))<\alpha(D)$ for each bounded $D \subset \operatorname{dom}(\mathrm{A})$ with $\alpha(D) \neq 0$, then $A$ is called condensing. It is easily seen that a compact map is 0 -set-contraction. We refer to [3], [4] for details and more properties.

Let $K$ be a closed convex set. For $x \in K$, let $I_{K}(x)=\{x+c(z-x): z \in$ $K$ and $c \geq 0\}$. Geometrically, it is the union of all rays beginning at $x$ and passing through some other points of $K$.

Definition 1 ([4, 11]). A map $A: \Omega \subset K \rightarrow X$ is said to be inward (respectively, weakly inward) on $\Omega$ relative to $K$ if $A x \in I_{K}(x)$ (respectively, $\left.A x \in \bar{I}_{K}(x)\right)$ for $x \in \Omega$, where $\bar{I}_{K}(x)$ denotes the closure of $I_{K}(x)$.

We refer to Deimling [4, Sections 18.3 and 20.4] for more details. Recall that $K$ is called a wedge if $\lambda x \in K$ for $x \in K$ and $\lambda \geq 0$. If a wedge $K$ also satisfies $K \cap(-K)=\{\theta\}$, then $K$ is called a cone.

Lemma 1. With respect to $\bar{I}_{K}(x)$, we have the following properties.

(1) $\bar{I}_{K}(x)$ is a closed convex set containing $K$ for each $x \in K$;

(2) If $K$ is a cone or a wedge in $X$, then $\bar{I}_{K}(x)$ is a wedge for each $x \in K$;

(3) If $K$ is a starred closed convex set, i.e., $x \in K$ implies $t x \in K$ for $t \in[0,1]$, then $\bar{I}_{K}(x)$ is also starred.

Proof. We only prove (3). With respect to (1) and (2), we refer to Lan and Webb [12]. Let $w=\lim _{n \rightarrow \infty} w_{n}$, where $w_{n}=x+c_{n}\left(y_{n}-x\right), y_{n} \in K, c_{n} \geq 0$. For each $t \in[0,1)$, we have

$$
\begin{aligned}
t w_{n} & =t x+t c_{n}\left(y_{n}-x\right) \\
& =x+t c_{n} y_{n}-\left(1-t+t c_{n}\right) x \\
& =x+\left(1-t+t c_{n}\right)\left(\frac{t c_{n}}{1-t+t c_{n}} y_{n}-x\right) .
\end{aligned}
$$

Since $0 \leq \frac{t c_{n}}{1-t+t c_{n}}<1$, so we have $\frac{t c_{n}}{1-t+t c_{n}} y_{n} \in K$, which implies $t w_{n} \in I_{K}(x)$ so that $t w \in \bar{I}_{K}(x)$ for each $t \in[0,1]$. 
Weakly inward mappings have been well studied by many authors. Among these literatures, Lan and Webb [11] has introduced the concept of generalized inward mappings which is a proper generalization of weakly inward mappings, i.e., all weakly inward mappings are generalized inward, but the converse does not hold (see Example 2.4 in [11]). It is the first purpose of this paper to introduce the fixed point of weakly inward 1-set-contraction mappings. Now we recall some definitions and results due to Lan and Webb [11].

Definition 2. Let $K$ be a closed convex set. A map $A: \Omega \subset K \rightarrow X$ is said to be generalized inward on $\Omega$ relative to $K$ if the following condition is satisfied:

$$
d(A x, K)<\|x-A x\| \text { for } x \in \Omega \text { with } A x \notin K,
$$

where $d(y, K)=\inf \{\|y-u\|: u \in K\}$.

Definition 3. A map $r$ from $X$ to $K$ is called a metric projection if $r$ satisfies $\|x-r x\|=d(x, K)$ for each $x \in X$.

Definition 4. $K$ is said to be an $M_{l}$-set for some $1 \leq l<\infty$ if there exists a continuous metric projection $r$ from $X$ to $K$ such that $\alpha(r)=l$, where $\alpha(r)=\inf \{k: r$ is a $k$-set-contraction map $\} . K$ is called an $M_{\infty}$-set if the metric projection is only continuous.

Lemma 2 ([11, Examples 2.6, 2.7]). If $X$ is a Hilbert space, then any closed convex set $K$ in $X$ is an $M_{1}$-set. And in any Banach space $X$, let $K$ be a ball, then $K$ is an $M_{1}$-set.

Now let $D$ be a bounded open set in $X$, let $K$ be a closed convex set and suppose that $D_{K}=D \cap K \neq \emptyset$. Denote by $\bar{D}_{K}$ the closure and $\partial D_{K}$ the boundary of $D_{K}$ relative to $K$.

From Definition 2.14 and Theorem 2.15 in Lan and Webb [11], we have the following lemma.

Lemma 3. Let $K$ be a closed convex $M_{1}$-set in a Banach space $X$ and $D$ a bounded open set in $X$ such that $D_{K} \neq \emptyset$. Assume that $A: \overline{D_{K}} \rightarrow X$ is a generalized inward $k$-set-contraction map with $k<1$ and $x \neq A x$ for $x \in \partial D_{K}$. Then there exists the fixed point index $i_{K}\left(A, D_{K}\right)$ satisfying the following properties.

$\left(P_{1}\right)$ (Solution property) If $i_{K}\left(A, D_{K}\right) \neq 0$, then $A$ has a fixed point in $D_{K}$.

$\left(P_{2}\right)$ (Normalization) If $u \in D_{K}$, then $i_{K}\left(\bar{u}, D_{K}\right)=1$, where $\bar{u}(x)=u$ for $x \in \overline{D_{K}}$.

$\left(P_{3}\right)$ (Additivity property) If $D_{1}, D_{2}$ are disjoint relatively open subsets of $D_{K}$ such that $x \neq A x$ for $x \in \overline{D_{K}} \backslash\left(D_{1} \cup D_{2}\right)$, then

$$
i_{K}\left(A, D_{K}\right)=i_{K}\left(A, D_{1}\right)+i_{K}\left(A, D_{2}\right) .
$$

$\left(P_{4}\right)$ (Homotopy property) If $H:[0,1] \times \overline{D_{K}} \rightarrow X$ is continuous and for each $t \in[0,1], H(t, \cdot): \partial D_{K} \rightarrow X$ is a generalized inward map. And 


$$
\begin{aligned}
& \alpha(H([0,1] \times D))<\alpha(D) \text { for each } D \subset \overline{D_{K}} \text { with } \alpha(D) \neq 0 \text {. If } x \neq \\
& H(t, x) \text { for } x \in \partial D_{K} \text { and } t \in[0,1] \text {, then } \\
& i_{K}\left(H(0, \cdot), D_{K}\right)=i_{K}\left(H(1, \cdot), D_{K}\right) .
\end{aligned}
$$

Remark 1. Since all weakly inward mappings are generalized inward (see [11, Lemma 2.3]), the fixed point $i_{K}\left(A, D_{K}\right)$ is also well-defined and has the above properties for the weakly inward $k$-set-contraction map $A$ with $k<1$.

Definition 5 ([14]). $A: \operatorname{dom}(\mathrm{A}) \subset \mathrm{X} \rightarrow \mathrm{X}$ is called a semiclosed 1-setcontraction mapping, if $A$ is 1-set-contraction and $I-A$ is closed.

Definition 6. A weakly inward mapping $A: \overline{D_{K}} \rightarrow X$ is said to satisfy $(C)$ condition, if $t A: \overline{D_{K}} \rightarrow X$ is also weakly inward for every $t \in[0,1]$.

Remark 2. Throughout this paper, we suppose that a weakly inward mapping $A: \overline{D_{K}} \rightarrow X$ satisfies $(C)$ condition. However, by Lemma 1 , it is easy to see that $(C)$ condition is satisfied automatically under one of the following conditions: (1) $\theta \in K$; (2) $K$ is a wedge; (3) $K$ is starred.

Next we introduce the main definition in this paper.

Definition 7. Let $K$ be a closed convex $M_{1}$-set in a Banach space $X$. Suppose that $A: \overline{D_{K}} \rightarrow X$ is a weakly inward 1-set-contraction mapping and $\theta \notin$ $\overline{(I-A) \partial D_{K}}$, then there exists $\delta>0$ such that

$$
\inf _{x \in \partial D_{K}}\|x-A x\| \geq \delta .
$$

Set $A_{k}=k A$, where $k \in\left(1-\frac{\delta}{M}, 1\right)$ and $M=\sup _{x \in \overline{D_{K}}}\|A x\|+\delta$. Then we define the fixed point index of $A$ over $D_{K}$ with respect to $K$ by

$$
i_{K}\left(A, D_{K}\right)=i_{K}\left(A_{k}, D_{K}\right) .
$$

It is readily checked that $i_{K}\left(A, D_{K}\right)$ makes sense and is independent of the choice of $A_{k}$. In fact, the weakly inwardness of $A$ and $(C)$ condition imply the weakly inwardness of $A_{k}$ which is also strict-set-contraction. Now suppose that $A_{k_{i}}: \overline{D_{K}} \rightarrow X$ are $k_{i}$-set-contraction mappings $\left(0<k_{i}<1\right)$ with

$$
\left\|A_{k_{i}} x-A x\right\|<\delta \quad \text { for } x \in \partial D_{K} \text { and } i=1,2 .
$$

Set $H(t, x)=t A_{k_{1}} x+(1-t) A_{k_{2}} x$. Clearly, $H:[0,1] \times \overline{D_{K}} \rightarrow X$ is continuous and $\alpha(H([0,1] \times D))<\alpha(D)$ for each $D \subset \overline{D_{K}}$ with $\alpha(D) \neq 0$. By Lemma 1 , $H(t, x) \in \bar{I}_{K}(x)$ for each $t \in[0,1]$, which shows that $H(t, \cdot): \partial D_{K} \rightarrow X$ is a weakly inward mapping for each $t \in[0,1]$. For $x \in \partial D_{K}$ and $t \in[0,1]$, we obtain

$$
\begin{aligned}
\|x-H(t, x)\| & =\left\|x-t A_{k_{1}} x-(1-t) A_{k_{2}} x\right\| \\
& \geq\|x-A x\|-t\left\|A x-A_{k_{1}} x\right\|-(1-t)\left\|A x-A_{k_{2}} x\right\| \\
& >\delta-t \delta-(1-t) \delta=0 .
\end{aligned}
$$


Then, by Lemma $3\left(P_{4}\right)$, we have

$$
i_{K}\left(A_{k_{1}}, D_{K}\right)=i_{K}\left(H(1, x), D_{K}\right)=i_{K}\left(H(0, x), D_{K}\right)=i_{K}\left(A_{k_{2}}, D_{K}\right),
$$

which implies that $i_{K}\left(A, D_{K}\right)$ is independent of $A_{k}$.

Remark 3 . It should be noted that $(C)$ condition in the above definition can be omitted if $K$ is in any case of (1)-(3) in Remark 2.

Theorem 1. Let $K$ be a closed convex $M_{1}$-set in a Banach space $X$ and $D$ a bounded open set in $X$ such that $D_{K} \neq \emptyset$. Suppose that $A: \overline{D_{K}} \rightarrow X$ is a weakly inward semiclosed 1-set-contraction mapping and $\theta \notin(I-A) \partial D_{K}$. Then the index $i_{K}\left(A, D_{K}\right)$ satisfies properties $\left(P_{1}\right),\left(P_{3}\right)$ as in Lemma 3 and

$\left(P_{2}\right)$ Let $\bar{u}(x)=u$ for $x \in \overline{D_{K}}$, then

$$
i_{K}\left(\bar{u}, D_{K}\right)= \begin{cases}1, & \text { when } u \in D_{K}, \\ 0, & \text { when } u \notin D_{K} .\end{cases}
$$

$\left(P_{4}\right)$ (Homotopy invariance) Suppose that $H:[0,1] \times \overline{D_{K}} \rightarrow X$ is continuous and $H(t, \cdot): \partial D_{K} \rightarrow X$ is weakly inward for each $t \in[0,1]$. If the measure of non-compactness $\alpha(H([0,1] \times D)) \leq \alpha(D)$ for each $D \subset \overline{D_{K}}$ and $\theta \notin \overline{(I-H)\left([0,1] \times \partial D_{K}\right)}$, then

$$
i_{K}\left(H(0, \cdot), D_{K}\right)=i_{K}\left(H(1, \cdot), D_{K}\right) .
$$

Proof. $\left(P_{2}\right)$ and $\left(P_{3}\right)$ are trivial. Next we shall prove $\left(P_{1}\right)$ and $\left(P_{4}\right)$.

Proof of $\left(P_{1}\right)$. Let $k_{n} \in(0,1)$ and $k_{n} \rightarrow 1$ as $n \rightarrow \infty$. Set $A_{n}=k_{n} A$ : $\overline{D_{K}} \rightarrow X$, then $A_{n}$ is weakly inward and $k_{n}$-set-contraction $\left(k_{n}<1\right)$. Noting that $\sup _{x \in \overline{D_{K}}}\|A x\|<+\infty$, since

$$
\left\|A x-A_{n} x\right\|=\left\|A x-k_{n} A x\right\|=\left(1-k_{n}\right)\|A x\| \rightarrow 0(n \rightarrow \infty),
$$

then there exists $N>0$ such that

$$
\left\|A x-A_{n} x\right\|<\delta \text { for every } n>N,
$$

where $0<\delta<\inf _{x \in \partial D_{K}}\|x-A x\|$, noting that $(I-A) \partial D_{K}$ is a closed set. And so we have $\left\|x-A_{n} x\right\| \geq\|x-A x\|-\left\|A x-A_{n} x\right\|>\delta-\delta=0$ for each $x \in \partial D_{K}$. By Definition $7, i_{K}\left(A, D_{K}\right)=i_{K}\left(A_{n}, D_{K}\right) \neq 0$. It follows from $\left(P_{1}\right)$ in Lemma 3 that $A_{n}$ has a fixed point $x_{n} \in D_{K}$. Then for every $n>N$,

$$
\left\|x_{n}-A x_{n}\right\|=\left\|A_{n} x_{n}-A x_{n}\right\| \rightarrow 0 \text { as } n \rightarrow \infty \text {. }
$$

Since $A$ is a semiclosed mapping, $\theta \in(I-A) \overline{D_{K}}$, i.e., there exists $x_{0} \in \overline{D_{K}}$ such that $A x_{0}=x_{0}$. The fact that $x \neq A x$ for each $x \in \partial D_{K}$ implies that $x_{0} \in D_{K}$ with $x_{0}=A x_{0}$.

Proof of $\left(P_{4}\right)$. By assumptions, there exist $\delta>0$ and $M>0$ such that

$$
\|x-H(t, x)\| \geq \delta>0 \quad \text { for } t \in[0,1] \text { and } x \in \partial D_{K}
$$


and $\|H(t, x)\| \leq M$ for $t \in[0,1]$ and $x \in \overline{D_{K}}$. Let $k \in\left(1-\frac{\delta}{M}, 1\right)$, set $G(t, x)=k H(t, x):[0,1] \times \overline{D_{K}} \rightarrow X$. Since, for $t \in[0,1]$ and $x \in \overline{D_{K}}$,

$$
\|H(t, x)-G(t, x)\|=(1-k)\|H(t, x)\| \leq(1-k) M<\delta,
$$

so for every $t \in[0,1]$ and $x \in \partial D_{K}$, we have

$$
\|x-G(t, x)\| \geq\|x-H(t, x)\|-\|H(t, x)-G(t, x)\|>\delta-\delta>0 .
$$

By $\left(P_{4}\right)$ in Lemma 3 ,

$$
i_{K}\left(G(0, \cdot), D_{K}\right)=i_{K}\left(G(1, \cdot), D_{K}\right) .
$$

Also it follows from Definition 7 that

$$
i_{K}\left(H(0, \cdot), D_{K}\right)=i_{K}\left(H(1, \cdot), D_{K}\right) .
$$

Remark 4. It should be noted that, in $\left(P_{4}\right)$, if $H:[0,1] \times \overline{D_{K}} \rightarrow X$ is also semiclosed, then $\theta \notin \overline{(I-H)\left([0,1] \times \partial D_{K}\right)}$ if and only if $x \neq H(t, x)$ for $x \in \partial D_{K}$ and $t \in[0,1]$. One will see that the remark is convenient for later applications to fixed point theorems.

\section{Fixed point theorems for weakly inward maps}

In this section we shall obtain some new fixed point theorems and nonzero fixed point theorems by using the fixed point index developed in Section 2 . These results improve and extend many relevant recent works (cf. [6], [11], [12], [14], [17], [19], [22], [23]).

Theorem 2. Let $K$ be a closed convex $M_{1}$-set in a Banach space $X$ and $D$ be a bounded open set in $K$ with $D \neq \emptyset$. Suppose that $A: \bar{D} \rightarrow X$ is a weakly inward semiclosed 1-set-contraction mapping and $x_{0} \in D$ such that

$$
x \neq t A x+(1-t) x_{0} \quad \text { for all } x \in \partial D \text { and } t \in(0,1) . \quad(L-S)
$$

Then $A$ has a fixed point in $\bar{D}$, and if $x \neq A x$ for $x \in \partial D$, then $i_{K}(A, D)=1$.

Proof. Without loss of generality, we assume $x \neq A x$ for $x \in \partial D$. Let

$$
H(t, x)=t A x+(1-t) x_{0} \text { for } x \in \partial D \text { and } t \in[0,1] .
$$

By the hypotheses and Lemma $1, H(t, \cdot): \partial D \rightarrow X$ is a weakly inward mapping for each $t \in[0,1]$ and $x \neq H(t, x)$ for $x \in \partial D$ and $t \in[0,1]$. One readily see that $(I-H)$ is closed (also see [15]).

In fact, let $S$ be a closed subset of $[0,1] \times \bar{D}$ of the form $S=M \times P$, where $M$ and $P$ is a closed subset of $[0,1]$ and $\bar{D}$ respectively. Suppose that $y_{n} \in(I-H)(M \times P)$ such that $y_{n}$ converges to $y_{0}$ as $n \rightarrow \infty$. Next we shall prove that $y_{0} \in(I-H)(M \times P)$. Since $I(t, x)=x$ for $x \in \bar{D}, t \in[0,1]$ and $y_{n}=$ $(I-H)\left(t_{n}, x_{n}\right)$, where $\left(t_{n}, x_{n}\right) \in M \times P$, so we have $x_{n}-t_{n} A x_{n}-\left(1-t_{n}\right) x_{0} \rightarrow y_{0}$. 
With passing to a subsequence if necessary, we suppose that $t_{n} \rightarrow t_{0}$ as $n \rightarrow \infty$. Clearly, $t_{0} \in M, 0 \leq t_{0} \leq 1$. And so

$$
\begin{aligned}
\left(I-t_{0} A\right) x_{n} & =x_{n}-t_{0} A x_{n} \\
& =x_{n}-t_{n} A x_{n}-\left(1-t_{n}\right) x_{0}-t_{0} A x_{n}+t_{n} A x_{n}+\left(1-t_{n}\right) x_{0} .
\end{aligned}
$$

Hence

$$
\left(I-t_{0} A\right) x_{n} \rightarrow y_{0}+\left(1-t_{0}\right) x_{0} \quad \text { as } n \rightarrow \infty .
$$

Since $t_{0} A$ is a semiclosed $t_{0}$-set-contraction mapping, there exists $e_{0} \in P$ such that $y_{0}+\left(1-t_{0}\right) x_{0}=e_{0}-t_{0} A e_{0}$, i.e.,

$$
y_{0}=e_{0}-t_{0} A e_{0}-\left(1-t_{0}\right) x_{0} .
$$

Consequently, $y_{0} \in(I-H)(M \times P)$, i.e., $(I-H)$ is a closed mapping. In addition, it is not difficult to prove that $\alpha\left(H_{\lambda}([0,1] \times Q)\right) \leq \alpha(Q)$ for any $Q \subset \bar{D}$ (also see the proof in Theorem 3). Hence it follows from Theorem 1 that $i_{K}(A, D)=i_{K}\left(x_{0}, D\right)=1$, which implies that $A$ has a fixed point in D.

Remark 5. Theorem 2 is an improvement of Theorem 3.2 in [11], Theorem 3 in [14], Theorem 1 in [6] and Lemma 2.2 in [22], respectively.

Corollary 1. Let $X, K, A$ be the same as in Theorem 2. If $x_{0}=\theta \in D$ and $x \neq t A x$ for $x \in \partial D$ and $t \in(0,1]$, then $i_{K}(A, D)=1$ and $A$ has a fixed point in $D$.

As an immediate consequence of Corollary 1 and Lemma 2, we derive the following corollary.

Corollary 2. Let $K$ be a closed convex set in a Hilbert space $H$ and $D$ a bounded open set in $K$ with $\theta \in D$. If $A: \bar{D} \rightarrow H$ is a weakly inward semiclosed 1-set-contraction mapping and

$$
x \neq t A x \quad \text { for } x \in \partial D \quad \text { and } t \in(0,1) .
$$

Then $A$ has a fixed point in $\bar{D}$.

Remark 6 . The above results still hold under the following boundary condition (see [11]):

$\left(B_{1}\right)\|A x\|<\|x\|+\|x-A x\|$ for each $x \in \partial D$ with $\|A x\|>\|x\|$.

$\left(B_{2}\right)$ When $X$ is a Hilbert space, $(x, A x)<\|x\|\|A x\|$ for each $x \in \partial D$ with $\|A x\|>\|x\|$.

Theorem 3. Let $K$ be an $M_{1}$-wedge in a Banach space $X$ and $D$ be a bounded open set in $K$. Suppose that $A: \bar{D} \rightarrow X$ is a weakly inward semiclosed 1-setcontraction mapping and there exists $e \in K \backslash\{\theta\}$ such that

$$
x \neq A x+\lambda e \quad \text { for } \lambda \geq 0 \text { and } x \in \partial D .
$$

Then $i_{K}(A, D)=0$. 
Proof. Since $\bar{D}$ is bounded, there exists $\lambda_{0}>0$ such that $\lambda e \notin \bar{D}$ for every $\lambda \geq \lambda_{0}$. Set

$$
H_{\lambda}(t, x)=(1-t) A x+t \lambda e \quad \text { for } \lambda \geq \lambda_{0}, t \in[0,1] \text { and } x \in \bar{D} .
$$

I) Let $Q$ be a subset in $\bar{D}$ such that the measure of non-compactness $\alpha(Q) \neq$ 0 , then we have $\alpha\left(H_{\lambda}([0,1] \times Q)\right) \leq \alpha(Q)$. Indeed,

$$
H_{\lambda}(t, Q)=(1-t) A(Q)+t \lambda e \subset \operatorname{co}(A(Q) \cup\{\lambda e\}) \text { for all } t \in[0,1] .
$$

Since $H_{\lambda}(t, x):[0,1] \times \bar{D} \rightarrow X$ is bounded and uniformly continuous with respect to $t$, so we have

$$
\begin{aligned}
\alpha\left\{H_{\lambda}([0,1] \times Q)\right\} & =\max _{t \in[0,1]} \alpha\left(H_{\lambda}(t, Q)\right) \leq \alpha\left(\operatorname{co}\left(A(Q) \cup\left\{x_{0}\right\}\right)\right) \\
& =\alpha(A(Q)) \leq \alpha(Q) .
\end{aligned}
$$

II) It follows from Lemma 1 that $H_{\lambda}(t, \cdot): \partial D \rightarrow X$ is a weakly inward mapping for each $t \in[0,1]$. Moreover, it is easy to verify that $H_{\lambda}$ is semiclosed (also see the proof in Theorem 2). Next we prove that $H_{\lambda}(t, x) \neq x$ for $x \in$ $\partial D, t \in[0,1]$ and $\lambda \geq \lambda_{0}$. In fact, suppose that it is not true. Then there exist $\lambda_{n}\left(\lambda_{n} \rightarrow \infty\right), x_{n} \in \partial D$ and $t_{n} \in[0,1]$ such that

$$
x_{n}=\left(1-t_{n}\right) A x_{n}+t_{n} \lambda_{n} e .
$$

Since the boundedness of the set $\left\{x_{n}-\left(1-t_{n}\right) A x_{n}\right\}$ implies that the number set $\left\{t_{n} \lambda_{n}\right\}$ is bounded, without loss of generality we suppose that $\lambda_{n} t_{n} \rightarrow t_{0}<$ $+\infty$, which shows that $t_{n} \rightarrow 0(n \rightarrow \infty)$, and so

$$
\begin{aligned}
x_{n}-A x_{n} & =\left(x_{n}-\left(1-t_{n}\right) A x_{n}\right)-t_{n} A x_{n} \\
& =t_{n} \lambda_{n} e-t_{n} A x_{n} \rightarrow t_{0} e(n \rightarrow \infty) .
\end{aligned}
$$

Since $I-A$ is closed, there exists $x \in \partial D$ such that $x-A x=t_{0} e$, which contradicts our hypothesis. It follows from Theorem 1 and $\lambda e \notin \bar{D}\left(\lambda \geq \lambda_{0}\right)$ that

$$
i_{K}(A, D)=i_{K}\left(H_{\lambda}(0, \cdot), D\right)=i_{K}\left(H_{\lambda}(1, \cdot), D\right)=i_{K}\left(\lambda x_{0}, D\right)=0 .
$$

Remark 7. Theorem 3 is an improvement of Theorem 4.1 in [11] and Theorem 4 in [14], respectively.

As a further consequence of Theorem 2 and Theorem 3, we can establish the following theorem when $K$ is a wedge.

Theorem 4. Let $K$ be an $M_{1}$-wedge in a Banach space $X$ and $D_{1}, D_{2}$ bounded open sets in $K$ with $\theta \in D_{1} \subset \overline{D_{1}} \subset D_{2}$. Suppose that $A: \overline{D_{2}} \rightarrow X$ is a weakly inward semiclosed 1-set-contraction mapping and the following conditions hold:

(1) There exists $x_{1} \in D_{1}$ such that $x \neq t A x+(1-t) x_{1}$ for $x \in \partial D_{1}$ and $t \in(0,1)$.

(2) There exists $e \in K \backslash\{\theta\}$ such that $x \neq A x+\lambda$ e for $x \in \partial D_{2}$ and $\lambda \geq 0$. 
Then $A$ has a fixed point in $\overline{D_{2}} \backslash D_{1}$.

Proof. We assume without loss of generality that $A$ has no fixed point on $\partial D_{1} \cup \partial D_{2}$. It follows from Theorem 2 that $i_{K}\left(A, D_{1}\right)=1$ and from Theorem 3 that $i_{K}\left(A, D_{2}\right)=0$. By the additivity property in Theorem 1 , we obtain

$$
i_{K}\left(A, D_{2} \backslash \overline{D_{1}}\right)=i_{K}\left(A, D_{2}\right)-i_{K}\left(A, D_{1}\right)=0-1=-1 .
$$

And thus there exists $x_{0} \in \overline{D_{2}} \backslash D_{1}$ such that $A x_{0}=x_{0}$.

Remark 8. The same assertion can be obtained when the hypotheses (1) and (2) are interchanged, i.e., (1) holds on $\partial D_{2}$ while (2) holds on $\partial D_{1}$. Moreover, Theorem 4 improves Theorem 4.2 in [11], Theorem 5 in [14] and Theorem 2 in $[6]$.

Let $K$ be a cone in Banach space $X$. Set $K_{r}=\{x \in K\|\| x \|<r\}, \partial K_{r}=$ $\{x \in K\||| x\|=r\}$ and $K_{r_{1}, r_{2}}=\left\{x \in K \mid r_{1}<\|x\|<r_{2}\right\}$. As an immediate consequence of Theorem 4 , we have the following corollaries.

Corollary 3. Let $K$ be an $M_{1}$-cone in a Banach space $X$ and $A: \overline{K_{r}} \rightarrow X$ a weakly inward semiclosed 1-set-contraction mapping. If there exist $r_{1}, r_{2}: 0<$ $r_{1}<r_{2}<r$ such that

(1) $x \neq t A x$ for each $x \in \partial K_{r_{2}}$ and $t \in(0,1]$;

(2) There exists $e \in K \backslash\{\theta\}$ such that $x-A x \neq \lambda$ e for $x \in \partial K_{r_{1}}$ and $\lambda \geq 0$. Then $A$ has a fixed point in $K_{r_{1}, r_{2}}$.

Corollary 4. Let $K$ be an $M_{1}$-cone in a Banach space $X$ and $A: \overline{K_{r}} \rightarrow X$ a weakly inward semiclosed 1-set-contraction mapping. If there exist $r_{1}, r_{2}$ : $0<r_{1}<r_{2}<r$ such that one of the following conditions holds

(1) $x \in \partial K_{r_{2}} \Rightarrow A x \nsupseteq x ; x \in \partial K_{r_{1}} \Rightarrow x \nsupseteq A x$,

(2) $x \in \partial K_{r_{1}} \Rightarrow A x \nsupseteq x ; x \in \partial K_{r_{2}} \Rightarrow x \nsupseteq A x$.

Then $A$ has a fixed point in $K_{r_{1}, r_{2}}$.

Theorem 5. Let $K$ be a closed convex $M_{1}$-set in a Banach space $X$. If $A$ : $K \rightarrow X$ is a weakly inward semiclosed 1-set-contraction mapping and $A(K)$ is bounded. Then there exists $\rho_{0}>0$ such that $i_{K}\left(A, B_{K}(\rho)\right)=1$ for all $\rho>\rho_{0}$, where $B(\rho)=\{x \in X:\|x\|<\rho\}$ and $B_{K}(\rho)=B(\rho) \cap K$. And so, A has a fixed point in $K$.

Proof. Since $A(K)$ is bounded, $A_{n}(K) \equiv k_{n} A(K)$ is also bounded for $k_{n} \in$ $(0,1)$ with $k_{n} \rightarrow 1(n \rightarrow \infty)$, which then implies that there exists $\rho_{0}>0$ such that $\overline{A(K) \cup A_{n}(K)} \subset B\left(\rho_{0}\right)$ for sufficiently large $n$. And so, for all $\rho>\rho_{0}$, $\overline{A_{n}(K)} \subset B(\rho), x \neq A_{n} x$ and $x \neq A x$ for $x \in \partial B_{K}(\rho)$. By Definition 7 and Theorem 3.1 in [11], we obtain

$$
i_{K}\left(A, B_{K}(\rho)\right)=i_{K}\left(A_{n}, B_{K}(\rho)\right)=1 .
$$

So $A$ has a fixed point in $B_{K}(\rho)$. 
Remark 9. Theorem 5 improves Theorem 3.1 in [11] and Theorem $3.1\left(H_{2}\right)$, Corollary $3.1\left(I_{2}\right)$ and Theorem $3.2\left(J_{2}\right)$ in Liu [17], respectively.

We may combine Theorem 4 and Theorem 5 to obtain the existence of at least two nonzero fixed points in $K$ under appropriate conditions.

Theorem 6. Let $K, D_{1}, D_{2}$ be the same as in Theorem 4. Let $A: K \rightarrow X$ be a weakly inward semiclosed 1-set-contraction mapping and $A(K)$ bounded. Assume that (1) of Theorem 4 holds on $\partial D_{1}$ and (2) holds on $\partial D_{2}$. Then $A$ has at least two nonzero fixed points in $K$.

Proof. Since $A(K)$ is bounded, by Theorem 5 , there exists a bounded open set $D$ such that $\overline{D_{2}} \subset D$ and $i_{K}(A, D)=1$. If $A$ has no fixed point on $\partial D$, then it follows from $(2)$ and $\left(P_{3}\right)$ that

$$
i_{K}\left(A, D \backslash \overline{D_{2}}\right)=i_{K}(A, D)-i_{K}\left(A, D_{2}\right)=1-0=1 .
$$

Thus $A$ has a fixed point in $D \backslash \overline{D_{2}}$. If $x \neq A x$ for $x \in \partial D_{1}$ (otherwise, the assertion holds), it follows from Theorem 4 that $A$ has a fixed point in $D_{2} \backslash \overline{D_{1}}$.

Theorem 7. Let $K$ be an $M_{1}$-cone of a Banach space $X$ and the norm monotonically increasing with respect to $K$. Suppose that $A: \overline{K_{r, R}} \rightarrow X$ is a weakly inward $k$-set-contraction mapping $(0<k<1)$ which satisfies one of the following conditions

$\left(C_{1}\right) x \in \partial K_{r} \Rightarrow\|A x\| \leq\|x\| ; x \in \partial K_{R} \Rightarrow\|A x\| \geq\|x\|$,

$\left(C_{2}\right) x \in \partial K_{R} \Rightarrow\|A x\| \leq\|x\| ; x \in \partial K_{r} \Rightarrow\|A x\| \geq\|x\|$.

Then $A$ has a fixed point in $\overline{K_{r, R}}$, where $\overline{K_{r, R}}=\{x \in K \mid r \leq\|x\| \leq R\}$.

Proof. We only prove this theorem under $\left(C_{1}\right)$. The proof is similar under $\left(C_{2}\right)$. Let $s=\frac{1}{2}(r+R)$, we define operator $A_{n}$ as follows

$$
A_{n} x= \begin{cases}A_{n}^{(1)}=\left(1+\frac{\|x\|-s}{n(R-s)}\right) A x & \text { for } x \in K \text { and } s \leq\|x\| \leq R, \\ A_{n}^{(2)}=\left(1-\frac{s-\|x\|}{n(s-r)}\right) A x & \text { for } x \in K \text { and } r \leq\|x\|<s .\end{cases}
$$

Then $A_{n}$ is continuous, bounded and weakly inward. Consider the mapping

$$
B_{n} x=\frac{\|x\|}{n m} A x \quad \text { for } \quad x \in \overline{K_{r, R}},
$$

where $m=\frac{1}{2}(R-r)$. For a subset $\Omega$ in $\overline{K_{r, R}}$, we have

$$
\alpha\left(B_{n}(\Omega)\right) \leq \frac{2 R}{n m} \alpha(A(\Omega)) \leq \frac{2 R}{n m} k \alpha(\Omega),
$$

which implies that $B_{n}$ is a weakly inward $\left(\frac{2 R k}{m n}\right)$-set-contraction mapping. Note that $\frac{2 R k}{m n} \rightarrow 0(n \rightarrow \infty)$, so $B_{n}$ is a weakly inward strict-set-contraction mapping in $\overline{K_{r, R}}$ for sufficiently large $n$.

In addition, one can see that $A_{n}^{(1)}$ and $A_{n}^{(2)}$ are $\left(1+\frac{s+2 R}{m n}\right) k$-set-contraction mappings. Hence $A_{n}^{(1)}$ and $A_{n}^{(2)}$ are strict-set-contraction for sufficiently large 
$n$. Let $K_{1}=\{x \in K \mid s \leq\|x\| \leq R\}, K_{2}=\{x \in K \mid r \leq\|x\|<s\}$ and $\overline{K_{r, R}}=K_{1} \cup K_{2}$. Then (Note that $\Omega=\left(\Omega \cap K_{1}\right) \cup\left(\Omega \cap K_{2}\right)$ ) we have

$$
\begin{aligned}
\alpha\left(A_{n}(\Omega)\right) & =\alpha\left\{\left(A_{n}\left(\Omega \cap K_{1}\right)\right) \cup\left(A_{n}\left(\Omega \cap K_{2}\right)\right)\right\} \\
& =\alpha\left\{\left(A_{n}^{(1)}\left(\Omega \cap K_{1}\right)\right) \cup\left(A_{n}^{(2)}\left(\Omega \cap K_{2}\right)\right)\right\} \\
& \leq\left(1+\frac{s+2 R}{m n}\right) k \alpha(\Omega),
\end{aligned}
$$

which implies that $A_{n}$ is also strict-set-contraction for sufficiently large $n$.

On the other hand, if there exists $x_{0} \in \partial K_{r}$ such that $A_{n} x_{0} \geq x_{0}$, it follows from hypotheses that

$$
r=\left\|x_{0}\right\| \leq\left\|A_{n} x_{0}\right\|=\left(1-\frac{1}{n}\right)\left\|A x_{0}\right\| \leq\left(1-\frac{1}{n}\right)\left\|x_{0}\right\|=\left(1-\frac{1}{n}\right) r<r .
$$

This contradiction shows that $A_{n} x \geq x$ for $x \in \partial K_{r}$. Similarly, $A_{n} x \not \leq x$ for $x \in \partial K_{R}$. By Corollary 4, there exists $x_{n} \in K_{r, R}$ such that $A x_{n}=x_{n}$. Without loss of generality we may assume that a subsequence $\left\{x_{n_{k}}\right\}$ of $\left\{x_{n}\right\}$ is in $K_{1}$. Since $A$ is strict-set-contraction, the set $\left\{\left\|A x_{n}\right\|\right\}$ is bounded. And so

$$
\begin{aligned}
\left\|x_{n_{k}}-A_{n} x_{n_{k}}\right\| & =\left\|x_{n_{k}}-\left(1+\frac{\left\|x_{n_{k}}\right\|-s}{n_{k}(R-s)}\right) A x_{n_{k}}\right\| \\
& =\frac{\left\|x_{n_{k}}\right\|-s}{n_{k}(R-s)}\left\|A x_{n_{k}}\right\| \leq \frac{1}{n_{k}}\left\|A x_{n_{k}}\right\| \rightarrow 0\left(n_{k} \rightarrow \infty\right) .
\end{aligned}
$$

Since a strict-set-contraction mapping is semiclosed, with passing to a subsequence if necessary, we may suppose that $x_{n_{k}} \rightarrow x^{*}$ as $n \rightarrow \infty$. By the definition of $A_{n}$, we obtain $x^{*} \in \overline{K_{r, R}}$ and $A x^{*}=x^{*}$.

Remark 10. Theorem 7 improves Theorem 2.1 in [23], lemma in [14] and some results due to Guo and Lakshmikantham [8], respectively.

Theorem 8. Let $K$ be an $M_{1}$-cone of Banach space $X$ and the norm monotone with respect to $K$. Suppose that $A: \overline{K_{r, R}} \rightarrow X$ is a weakly inward semiclosed 1-set-contraction mapping and there exists $\delta>0$ such that

$\left(H_{1}\right) x \in \partial K_{r} \Rightarrow\|A x\| \leq\|x\| ; x \in \partial K_{R} \Rightarrow\|A x\| \geq(1+\delta)\|x\|$, or

$$
\left(H_{2}\right) x \in \partial K_{R} \Rightarrow\|A x\| \leq\|x\| ; x \in \partial K_{r} \Rightarrow\|A x\| \geq(1+\delta)\|x\| .
$$

Then $A$ has a fixed point in $\overline{K_{r, R}}$.

Proof. Since $A: \overline{K_{r, R}} \rightarrow X$ is a 1-set-contraction mapping, then $\alpha(A(\Omega)) \leq$ $\alpha(\Omega)$ for an open subset $\Omega \subset \overline{K_{r, R}}$. Now consider the mapping $A_{n}$ as follows

$$
A_{n} x=\lambda_{n} A x, \quad \text { where } \quad \lambda_{n}=\frac{n-1}{n} .
$$

Then $\alpha\left(A_{n}(\Omega)\right)=\frac{n-1}{n} \alpha(A(\Omega)) \leq \frac{n-1}{n} \alpha(\Omega)$, which implies that $A_{n}$ is strictset-contraction. And it is easy to see that $A_{n}$ is weakly inward. 
If $\left(H_{1}\right)$ is satisfied, noting that $\frac{1}{1+\delta}<\lambda_{n}<1$ when $n$ is sufficiently large, then we get

$$
\left\|A_{n} x\right\|=\lambda_{n}\|A x\|<\|A x\| \leq\|x\| \quad \text { for } x \in \partial K_{r}
$$

and

$$
\left\|A_{n} x\right\|=\lambda_{n}\|A x\|>\frac{1}{1+\delta}\|A x\| \geq\|x\| \quad \text { for } x \in \partial K_{R} .
$$

It follows from Theorem 7 that there exists $x_{n} \in \overline{K_{r, R}}$ such that $x_{n}=A_{n} x_{n}=$ $\lambda_{n} A x_{n}$. And thus

$$
x_{n}-A x_{n}=\lambda_{n} A x_{n}-A x_{n}=\left(\lambda_{n}-1\right) A x_{n} \rightarrow \theta \quad \text { as } n \rightarrow \infty .
$$

Since $A: \overline{K_{r, R}} \rightarrow X$ is semiclosed, there exists $x_{0} \in \overline{K_{r, R}}$ such that $A x_{0}=x_{0}$.

Similarly, the same assertion remains valid under $\left(H_{2}\right)$.

Remark 11. Theorem 8 improves Theorem 7 in [14] and Corollary 2.1, Theorem 2.2 and Corollary 2.2 in [23], respectively.

\section{Multiple positive fixed points}

Leggett and Williams [13] has obtained multiple fixed points of completely continuous operators that are self-maps defined on a cone. By virtue of the index introduced in [20], Sun and Sun have obtained the same assertion for weakly inward and completely continuous operators under appropriate conditions. In this section, we shall prove that the same results remain valid for weakly inward semiclosed 1-set-contraction mappings. For the sake of convenience, we first recall a similar concept due to Amann [1] (also see [8]). A non-negative continuous functional $f(x)$ defined on a cone $P$ is said to be concave if

$$
f(t x+(1-t) y) \geq t f(x)+(1-t) f(y) \quad \text { for } x, y \in P \text { and } t \in[0,1] .
$$

Let $P(f, a, b)=\{x \in P: a \leq f(x)$ and $\|x\| \leq b\}$ for $0<a<b$. Evidently, $P(f, a, b)$ is a bounded closed convex set.

Lemma 4. Let $K$ be a bounded closed convex $M_{1}$-set in a Banach space $X$ and $A: K \rightarrow X$ a weakly inward semiclosed 1-set-contraction mapping. Then $i_{K}(A, K)=1$.

Proof. Since $K$ is open and $\partial K=\emptyset$ relative to $K, i_{K}(A, K)$ makes sense. For a fixed element $v \in K$, let $H(t, x)=t v+(1-t) A x$ for $x \in K$ and $t \in[0,1]$. From $\partial K=\emptyset$, it follows that $H(t, x) \neq x$ for $x \in \partial K$ and $t \in[0,1]$, and so

$$
i_{K}(A, K)=i_{K}(v, K)=1 .
$$

This completes the proof.

Now we introduce the main theorem of this section. 
Theorem 9. Let $P$ be a cone in a real Banach space $X$ and $A: \overline{P_{c}} \rightarrow X$ a weakly inward (relative to $\overline{P_{c}}$ ) semiclosed 1-set-contraction mapping, where $\overline{P_{c}}=\{x \in P:\|x\| \leq c\}$. Suppose that there exists a non-negative continuous concave functional $f(x)$ defined on $P$ such that $f(x) \leq\|x\|$ for $x \in \overline{P_{c}}$. If there exist $0<d<a<b \leq c$ such that

(1) $\{x \in P(f, a, b): f(x)>a\} \neq \emptyset$, and $f(A x)>a$ whenever $x \in P(f, a, b)$;

(2) $x \neq t A x$ for $x \in \partial P_{d}$ and $t \in(0,1]$;

(3) $f(A x)>a$ for $x \in P(f, a, c)$ with $\|A x\|>b$.

Then $A$ has at least three fixed points in $\overline{P_{c}}$.

Proof. Set $U_{1}=\left\{x \in \overline{P_{c}}:\|x\|<d\right\}$ and $U_{2}=\{x \in P(f, a, c)$ and $f(x)>a\}$. It easily follows from $f(x) \leq\|x\|$ that $U_{1}, U_{2}$ are two disjoint nonempty bounded open sets relative to $\overline{P_{c}}$. Now we take $\overline{P_{c}}$ as $K$ in Definition 7 , i.e., write $K \equiv \overline{P_{c}}$, then it follows from Lemma 2 that $K$ is a closed convex $M_{1}$-set. By (2) and Corollary 1, we have

$$
i_{K}\left(A, U_{1}\right)=1 \text {. }
$$

Now we claim that $A x \neq x$ for $x \in \partial U_{2}$. In fact, suppose that it is not true. Then there exists $x_{0} \in \partial U_{2}$ such that $A x_{0}=x_{0}$, and hence $f\left(x_{0}\right)=a$ and either $x_{0} \in P(f, a, b)$ or $\left\|x_{0}\right\|>b$. If $x_{0} \in P(f, a, b)$, it follows from (1) that $f\left(x_{0}\right)=f\left(A x_{0}\right)>a$, a contradiction. If $\left\|x_{0}\right\|>b$, we have $\left\|A x_{0}\right\|=\left\|x_{0}\right\|>b$. So it follows from $(3)$ that $f\left(x_{0}\right)=f\left(A x_{0}\right)>a$, a contradiction with $f\left(x_{0}\right)=a$. Hence $A x \neq x$ for all $x \in \partial U_{2}$, which shows that $i_{K}\left(A, U_{2}\right)$ makes sense.

By (1), let $v \in P(f, a, b)$ with $f(v)>a$. And we set

$$
H(t, x)=t v+(1-t) A x \quad \text { for } x \in \overline{U_{2}} \text { and } t \in[0,1] .
$$

As in former sections, it is easy to verify that $H:[0,1] \times \overline{U_{2}} \rightarrow X$ is continuous, semiclosed and $H(t, \cdot): \partial U_{2} \rightarrow X$ is weakly inward for each $t \in$ $[0,1]$ with $\alpha(H([0,1] \times D)) \leq \alpha(D)$ for any $D \subset \overline{U_{2}}$. Suppose that there exist $t_{0} \in[0,1]$ and $x_{0} \in \partial U_{2}$ such that $H\left(t_{0}, x_{0}\right)=x_{0}$, then $f\left(x_{0}\right)=a$. If $\left\|A x_{0}\right\|>b$, it follows from $(3)$ that $f\left(A x_{0}\right)>a$, and so

$$
\begin{aligned}
f\left(x_{0}\right) & =f\left(H\left(t_{0}, x_{0}\right)\right)=f\left(t_{0} v+\left(1-t_{0}\right) A x_{0}\right) \\
& \geq t_{0} f(v)+\left(1-t_{0}\right) f\left(A x_{0}\right)>a,
\end{aligned}
$$

which is a contradiction to $f\left(x_{0}\right)=a$. On the other hand, if $\left\|A x_{0}\right\| \leq b$, then we have

$$
\begin{aligned}
\left\|x_{0}\right\| & =\left\|t_{0} v+\left(1-t_{0}\right) A x_{0}\right\| \\
& \leq t_{0}\|v\|+\left(1-t_{0}\right)\left\|A x_{0}\right\| \leq b,
\end{aligned}
$$

which implies that $x_{0} \in P(f, a, b)$. Therefore it follows from (1) that $f\left(A x_{0}\right)>$ $a$. As the same in the first case, we also get a contradiction. Thus $H(t, x) \neq x$ for each $x \in \partial U_{2}$ and $t \in[0,1]$. By virtue of $\left(P_{4}\right)$ and $\left(P_{2}\right)$ in Theorem 1 , we obtain

$$
i_{K}\left(A, U_{2}\right)=i_{K}\left(H(0, \cdot), U_{2}\right)=i_{K}\left(H(1, \cdot), U_{2}\right)=i_{K}\left(v, U_{2}\right)=1 .
$$


From Lemma 4 and $\left(P_{3}\right)$ in Theorem 1, it follows that

$$
\begin{aligned}
i_{K}\left(A, K \backslash\left(\overline{U_{1} \cup U_{2}}\right)\right) & =i_{K}(A, K)-i_{K}\left(A, U_{1}\right)-i_{K}\left(A, U_{2}\right) \\
& =1-1-1=-1 .
\end{aligned}
$$

By $\left(P_{1}\right)$ in Theorem 1 , there exist $x_{1} \in U_{1}, x_{2} \in U_{2}$ and $x_{3} \in \overline{P_{c}} \backslash \overline{U_{1} \cup U_{2}}$ such that $A x_{i}=x_{i}(i=1,2,3)$.

\section{An eigenvalue problem}

In this section we discuss the eigenvalue problem:

$$
A x=\mu x \quad \text { for } \quad x \in K \backslash\{\theta\} \text { and } \mu>0 \text {, }
$$

which has been thoroughly studied when $A$ is a mapping from $K_{r}$ into $K$. However, to our best knowledge, very little is known when $A$ is weakly inward except Zou [23].

Theorem 10. Let $K$ be an $M_{1}$-cone in a Banach space $X$ and the norm monotone with respect to $K$. Suppose that $A: K \rightarrow X$ is a weakly inward semiclosed 1-set-contraction mapping and one of the following conditions holds

$$
\begin{array}{ll}
\lim _{\|x\| \rightarrow 0, x \in K} \frac{\|A x\|}{\|x\|}=\infty ; & \lim _{\|x\| \rightarrow \infty, x \in K} \frac{\|A x\|}{\|x\|}=\sigma \in[0,1), \\
\lim _{\|x\| \rightarrow \infty, x \in K} \frac{\|A x\|}{\|x\|}=\infty ; & \lim _{\|x\| \rightarrow 0, x \in K} \frac{\|A x\|}{\|x\|}=\sigma \in[0,1) .
\end{array}
$$

Then for each $\lambda \geq 1$, there exists $x_{\lambda} \in K \backslash\{\theta\}$ such that $A x_{\lambda}=\lambda x_{\lambda}$. Moreover, $\lim _{\lambda \rightarrow \infty}\left\|x_{\lambda}\right\|=0$ under (1) and $\lim _{\lambda \rightarrow \infty}\left\|x_{\lambda}\right\|=\infty$ under (2).

Proof. we only prove this theorem for the first case. Let $\lambda \geq 1$ be arbitrarily fixed and consider $\frac{1}{\lambda}$-set-contraction mapping $A_{\lambda}=\frac{1}{\lambda} A$. By hypothesis (1), there exist $r>0$ and $\delta>0$ such that

$$
\left\|A_{\lambda} x\right\| \geq(1+\delta)\|x\| \quad \text { for } \quad x \in \partial K_{r} .
$$

Also by (1), for each $\varepsilon>0$ with $\sigma+\varepsilon \leq 1$, there exists $R(>r)$ such that

$$
\frac{\left\|A_{\lambda} x\right\|}{\|x\|}=\frac{1}{\lambda} \frac{\|A x\|}{\|x\|} \leq \frac{\|A x\|}{\|x\|} \leq \sigma+\varepsilon \leq 1,
$$

i.e., $\left\|A_{\lambda} x\right\| \leq\|x\|$ for every $x \in \partial K_{R}$. Therefore, it follows from Theorem 8 that there exists $x_{\lambda} \in \overline{K_{r, R}}$ such that $A_{\lambda} x_{\lambda}=x_{\lambda}$, i.e., $A x_{\lambda}=\lambda x_{\lambda}$.

It remains to prove $\left\|x_{\lambda}\right\| \rightarrow 0$ as $\lambda \rightarrow \infty$. Suppose that it is not true. Then there exists $d(0<d \leq+\infty)$ such that $\left\|x_{\lambda_{n}}\right\| \rightarrow d$ as $\lambda_{n} \rightarrow \infty$ for some subsequence $\left\{x_{\lambda_{n}}\right\}$.

If $d<+\infty$, then $\frac{d}{2} \leq\left\|x_{\lambda_{n}}\right\| \leq 2 d$ for sufficiently large $n$, and hence

$$
\lambda_{n}=\frac{\left\|A x_{\lambda_{n}}\right\|}{\left\|x_{\lambda_{n}}\right\|} \leq \frac{2 M}{d},
$$

where $M=\sup _{\|x\| \leq 2 d}\|A x\|$, which contradicts $\lambda_{n} \rightarrow \infty$. 
If $d=+\infty$, then by hypothesis (1), we have

$$
\lambda_{n}=\frac{\left\|A x_{\lambda_{n}}\right\|}{\left\|x_{\lambda_{n}}\right\|} \rightarrow \tau \in[0,1),
$$

which contradicts $\lambda_{n} \rightarrow \infty$. So we obtain $\lim _{\lambda \rightarrow \infty}\left\|x_{\lambda}\right\|=0$.

Remark 12. Theorem 10 improves Theorem 3.1 in [23] and some relevant results obtained by Guo and Lakshmikantham [8], respectively.

Remark 13. We do not know whether the results established in this paper remain valid for generalized inward 1-set-contraction mappings.

Acknowledgement. The first author thanks Professor Yiming Long for constant encouragement and help.

\section{References}

[1] H. Amann, Fixed point equations and nonlinear eigenvalue problems in ordered Banach spaces, SIAM Rev. 18 (1976), no. 4, 620-709.

[2] J. Caristi, Fixed point theorems for mappings satisfying inwardness conditions, Trans. Amer. Math. Soc. 215 (1976), 241-251.

[3] J. Cronin, Eigenvalues of some nonlinear operators, J. Math. Anal. Appl. 38 (1972), 659-667.

[4] K. Deimling, Nonlinear Functional Analysis, Springer-Verlag, Berlin, 1985.

[5] _ Fixed points of weakly inward multis, Nonlinear Anal. 10 (1986), no. 11, 12611262.

[6] _ Positive fixed points of weakly inward maps, Nonlinear Anal. 12 (1988), no. 3, 223-226.

[7] K. Deimling and S. Hu, Fixed points of weakly inward maps in conical shells, Nonlinear Anal. 12 (1988), no. 3, 227-230.

[8] D. Guo and V. Lakshmikantham, Nonlinear Problems in Abstract Cones, Academic Press, Inc., Boston, MA, 1988.

[9] B. Halpern and G. Bergman, A fixed-point theorem for inward and outward maps, Trans. Amer. Math. Soc. 130 (1968), 353-358.

[10] S. Hu, K. Deimling, and J. Prub, Fixed points of weakly inward multivalued maps, Nonlinear Anal. 10 (1986), no. 5, 465-469.

[11] K. Lan and J. Webb, A fixed point index for generalized inward mappings of condensing type, Trans. Amer. Math. Soc. 349 (1997), no. 6, 2175-2186.

[12] _ A fixed point index for weakly inward A-proper maps, Nonlinear Anal. 28 (1997), no. 2, 315-325.

[13] R. Leggett and L. Williams, Multiple positive fixed points of nonlinear operators on ordered Banach spaces, Indiana Univ. Math. J. 28 (1979), no. 4, 673-688.

[14] G. Li, The fixed-point index and the fixed-point theorems of 1-set-contraction mappings, Proc. Amer. Math. Soc. 104 (1988), no. 4, 1163-1170.

[15] G. Li, S. Xu, and H. Duan, Fixed point theorems of 1-set-contractive operators in Banach spaces, Appl. Math. Lett. 19 (2006), no. 5, 403-412.

[16] L. Liu, Some random approximations and random fixed point theorems for 1-setcontractive random operators, Proc. Amer. Math. Soc. 125 (1997), no. 2, 515-521.

[17] _ Approximation theorems and fixed point theorems for various classes of 1-setcontractive mappings in Banach spaces, Acta Math. Sin. (Engl. Ser.) 17 (2001), no. 1, $103-112$. 
[18] I. Massabo and C. Stuart, Positive eigenvectors of $k$-set contractions, Nonlinear Anal. 3 (1979), no. 1, 35-44.

[19] W. Petryshyn, Remarks on condensing and k-set-contractive mappings, J. Math. Anal. Appl. 39 (1972), 717-741.

[20] Y. Sun and J. Sun, Multiple positive fixed points of weakly inward mappings, J. Math. Anal. Appl. 148 (1990), no. 2, 431-439.

[21] J. Webb, A-properness and fixed points of weakly inward mappings, J. London Math. Soc. (2) 27 (1983), no. 1, 141-149.

[22] S. Xu, B. Jia, and G. Li, Fixed points for weakly inward mappings in Banach spaces, J. Math. Anal. Appl. 319 (2006), no. 2, 863-873.

[23] W. Zou, Positive fixed point and eigenvalue results for weakly inward maps, Nonlinear Anal. 27 (1996), no. 12, 1447-1461.

Huagui Duan

School of Mathematical Science and LPMC

NANKAI UNIVERSITY

Tianjin 300071, P. R. China

E-mail address: duanhg@nankai.edu.cn

ShaOyUan Xu

School of Mathematics and Statistics

Hubei Normal University

HuAngshi 435002, P. R. China

E-mail address: xushaoyuan@126.com

GuOZHEN Li

School of Mathematics and Information Science

JiangXi Normal University

NANCHANG 330027 , P. R. China 